\title{
SOME COVERS AND ENVELOPES IN THE CHAIN COMPLEX CATEGORY OF $R$-MODULES
}

\author{
ZHANPING WANG ${ }^{凶}$ and ZHONGKUI LIU
}

(Received 3 October 2009; accepted 30 March 2011)

Communicated by J. Du

\begin{abstract}
We study the existence of some covers and envelopes in the chain complex category of $R$-modules. Let $(\mathcal{A}, \mathcal{B})$ be a cotorsion pair in $R$-Mod and let $\mathcal{E} \mathcal{A}$ stand for the class of all exact complexes with each term in $\mathcal{A}$. We prove that $\left(\mathcal{E} \mathcal{A}, \mathcal{E} \mathcal{A}^{\perp}\right)$ is a perfect cotorsion pair whenever $\mathcal{A}$ is closed under pure submodules, cokernels of pure monomorphisms and direct limits and so every complex has an $\mathcal{E} \mathcal{A}$-cover. As an application we show that every complex of $R$-modules over a right coherent ring $R$ has an exact Gorenstein flat cover. In addition, the existence of $\overline{\mathcal{A}}$-covers and $\overline{\mathcal{B}}$-envelopes of special complexes is considered where $\overline{\mathcal{A}}$ and $\overline{\mathcal{B}}$ denote the classes of all complexes with each term in $\mathcal{A}$ and $\mathcal{B}$, respectively.
\end{abstract}

2010 Mathematics subject classification: primary 18G35; secondary 55U15, 03 C35.

Keywords and phrases: cotorsion pair, cover, envelope, Gorenstein flat complex.

\section{Introduction}

In this paper $R$ denotes a ring with unity. We let $\mathscr{C}(R)$ denote the abelian category of complexes of left $R$-modules. A complex

$$
\ldots \stackrel{\delta^{-2}}{\longrightarrow} C^{-1} \stackrel{\delta^{-1}}{\longrightarrow} C^{0} \stackrel{\delta^{0}}{\longrightarrow} C^{1} \stackrel{\delta^{1}}{\longrightarrow} \cdots
$$

of left $R$-modules will be denoted by $(C, \delta)$ or $C$. For a left $R$-module $M$ we will use $\bar{M}$ to denote the complex

$$
\cdots \longrightarrow 0 \longrightarrow M \stackrel{\text { id }}{\longrightarrow} M \longrightarrow 0 \longrightarrow \cdots
$$

with $M$ in the -1 st and 0th positions in $R$-Mod. We denote by $\underline{M}$ and $M^{+}$the complex with $M$ in the 0 th place and 0 elsewhere, and the character module $\operatorname{Hom}_{\mathbb{Z}}(M, \mathbb{Q} / \mathbb{Z})$ respectively. Given a complex $C$ and an integer $m$, we denote by $C[m]$ the complex such that $C[m]^{n}=C^{m+n}$ and the boundary operators are $(-1)^{m} \delta^{m+n}$.

In this paper, we use both subscripts and superscripts. When we use superscripts for a complex, we use subscripts to distinguish positions within the complexes.

Supported by the National Natural Science Foundation of China (10961021).

(C) 2011 Australian Mathematical Publishing Association Inc. 1446-7887/2011 \$16.00 
For example, if $\left(K_{i}\right)_{i \in I}$ is a family of complexes, then $K_{i}^{n}$ denotes the degree- $n$ term of the complex $K_{i}$.

We denote by $\operatorname{Hom}(C, D)$ the abelian group of morphisms from $C$ to $D$ in $\mathscr{C}(R)$ and by $\operatorname{Ext}^{i}(C, D)$, where $i \geq 1$, the groups that we get from the right derived functor of Hom. We let $\mathcal{H} \operatorname{om}(C, D)$ denote the complex of abelian groups with

$$
\mathcal{H o m}(C, D)^{n}=\prod_{i \in \mathbb{Z}} \operatorname{Hom}_{R}\left(C^{i}, D^{n+i}\right)
$$

and

$$
\delta^{n}\left(\left(f^{i}\right)_{i \in \mathbb{Z}}\right)=\left(\delta^{n+i} f^{i}-(-1)^{n} f^{i+1} \delta^{i}\right)_{i \in \mathbb{Z}}
$$

for $\left(f^{i}\right)_{i \in \mathbb{Z}} \in \mathcal{H} \operatorname{om}(C, D)^{n}$.

Let $Z(-), B(-)$ and $H(-)$ denote the cycles, boundaries, and homology functors respectively. It is easy to see that

$$
\operatorname{Hom}(C, D)=Z^{0}(\mathcal{H} \operatorname{om}(C, D)) \text {. }
$$

General background material can be found in $[5-7,11,13]$.

Next we recall some known concepts and facts used in what follows. Let $\mathcal{A}$ and $\mathcal{B}$ be classes of objects in an abelian category $\mathscr{D}$ which has enough projectives and enough injectives. Let $D$ be an object of $\mathscr{D}$. We recall some definitions introduced in [4]. An object $B$ in $\mathcal{B}$ is called a $\mathcal{B}$-preenvelope of $D$ if there exists a homomorphism $\alpha: D \longrightarrow B$ such that the diagram

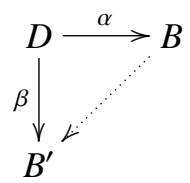

can be completed for each homomorphism $\beta: D \longrightarrow B^{\prime}$ with $B^{\prime}$ in $\mathcal{B}$. Furthermore, if the triangle

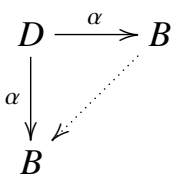

can be completed only by automorphisms, then we say that $\alpha: D \longrightarrow B$ is a $\mathcal{B}$-envelope.

A monomorphism $\alpha: D \longrightarrow B$ with $B \in \mathcal{B}$ is said to be a special $\mathcal{B}$-preenvelope of $D$ if $\operatorname{Coker}(\alpha) \in{ }^{\perp} \mathcal{B}$. A class $\mathcal{B}$ is called (pre)enveloping if every object of $\mathscr{D}$ has a $\mathcal{B}$-(pre)envelope. We also have the dual concepts of a (special) $\mathcal{B}$-precover, $\mathcal{B}$-cover and (pre)covering class.

In [1, Theorem 2.10] the authors proved that every module has an $\mathcal{A}$-cover whenever it has an $\mathcal{A}$-precover and $\mathcal{A}$ is closed under direct limits. A pair of classes of objects $(\mathcal{A}, \mathcal{B})$ is called a cotorsion pair or cotorsion theory (see [17, 22]) if $\mathcal{A}^{\perp}=\mathcal{B}$ and ${ }^{\perp} \mathcal{B}=\mathcal{A}$ where

$$
\mathcal{A}^{\perp}=\left\{B \in \mathscr{D} \mid \operatorname{Ext}^{1}(A, B)=0 \forall A \in \mathcal{A}\right\},
$$


and

$$
{ }^{\perp} \mathcal{B}=\left\{A \in \mathscr{D} \mid \operatorname{Ext}^{1}(A, B)=0 \forall B \in \mathcal{B}\right\} .
$$

A cotorsion pair $(\mathcal{A}, \mathcal{B})$ is called hereditary if whenever

$$
0 \longrightarrow A^{\prime} \longrightarrow A \longrightarrow A^{\prime \prime} \longrightarrow 0
$$

is exact with $A, A^{\prime \prime} \in \mathcal{A}$, then $A^{\prime}$ is also in $\mathcal{A}$. This is equivalent to the requirement that if whenever

$$
0 \longrightarrow B^{\prime} \longrightarrow B \longrightarrow B^{\prime \prime} \longrightarrow 0
$$

is exact with $B^{\prime}$ and $B \in \mathcal{B}$, then $B^{\prime \prime}$ is also in $\mathcal{B}$.

A cotorsion pair $(\mathcal{A}, \mathcal{B})$ is called complete if every $D \in \mathscr{D}$ has a special $\mathcal{B}$-preenvelope and a special $\mathcal{A}$-precover. A cotorsion pair $(\mathcal{A}, \mathcal{B})$ is called perfect if every $D \in \mathscr{D}$ has a $\mathcal{B}$-envelope and an $\mathcal{A}$-cover. A cotorsion pair $(\mathcal{A}, \mathcal{B})$ is said to be cogenerated by a set $X$ if $X^{\perp}=\mathcal{A}^{\perp}$.

It is well known that a perfect cotorsion pair is complete, but the converse may be false in general. In [3] Eklof and Trlifaj proved that a cotorsion pair $(\mathcal{A}, \mathcal{B})$ in $R$-Mod is complete when it is cogenerated by a set. This result actually holds in a Grothendieck category with enough projectives, as Hovey proved in [19]. For unexplained concepts and notation we refer the reader to [8, 13, 17, 24].

In [14] Gillespie introduced the following definition.

Definition 1.1 [14, Definition 3.3]. Let $(\mathcal{A}, \mathcal{B})$ be a cotorsion pair on an abelian category $\mathscr{C}$. Let $X$ be a chain complex.

(1) $X$ is called an $\mathcal{A}$ complex if it is exact and $Z^{n} X \in \mathcal{A}$ for all $n$.

(2) $X$ is called a $\mathcal{B}$ complex if it is exact and $Z^{n} X \in \mathcal{B}$ for all $n$.

(3) $X$ is called a $\operatorname{dg}-\mathcal{A}$ complex if $X^{n} \in \mathcal{A}$ for each $n$ and $\mathcal{H} \operatorname{om}(X, B)$ is exact whenever $B$ is a $\mathcal{B}$ complex.

(4) $X$ is called a $\operatorname{dg}-\mathcal{B}$ complex if $X^{n} \in \mathcal{B}$ for each $n$ and $\mathcal{H} \operatorname{om}(A, X)$ is exact whenever $A$ is an $\mathcal{A}$ complex.

We denote the class of $\mathcal{A}$ complexes by $\widetilde{\mathcal{A}}$ and the class of dg- $\mathcal{A}$ complexes by $\operatorname{dg} \widetilde{\mathcal{A}}$. Similarly, the class of $\mathcal{B}$ complexes is denoted by $\widetilde{B}$ and the class of $\operatorname{dg}-\mathcal{B}$ complexes is denoted by $\operatorname{dg} \widetilde{\mathcal{B}}$.

In [14] it was shown that $(\widetilde{\mathcal{A}}, \mathrm{dg} \widetilde{\mathcal{B}})$ and $(\mathrm{dg} \widetilde{\mathcal{A}}, \widetilde{\mathcal{B}})$ are cotorsion pairs in $\mathscr{C}(R)$ if $(\mathcal{A}, \mathcal{B})$ is a cotorsion pair in $R$-Mod. It is also proved that $(\mathcal{A}, \mathcal{B})$ is hereditary if and only if $(\widetilde{\mathcal{A}}, \operatorname{dg} \widetilde{\mathcal{B}})$ is hereditary or, equivalently, if $(\operatorname{dg} \widetilde{\mathcal{A}}, \widetilde{\mathcal{B}})$ is hereditary. But the question of whether or not the induced cotorsion pairs are complete when the original cotorsion pair is complete is open (see [14]).

Let $(\mathcal{A}, \mathcal{B})$ be a cotorsion pair in $R$-Mod. In [15, Proposition 3.8] Gillespie proved that the induced cotorsion pair $(\operatorname{dg} \widetilde{\mathcal{A}}, \widetilde{\mathcal{B}})$ is complete whenever $(\mathcal{A}, \mathcal{B})$ is cogenerated by a set. In [23] it was proved that the induced cotorsion pairs $(\widetilde{\mathcal{A}}, \operatorname{dg} \widetilde{\mathcal{B}})$ and $\left(\overline{\mathcal{A}}, \overline{\mathcal{A}}^{\perp}\right)$ are complete whenever $\mathcal{A}$ is closed under pure submodules and cokernels of pure 
monomorphisms. Here $\overline{\mathcal{A}}$ stands for the class of all complexes with each term in $\mathcal{A}$. In [16] it was shown that $\left(\mathcal{E} \mathcal{A}, \mathcal{E} \mathcal{A}^{\perp}\right)$ is a complete cotorsion pair whenever $\mathcal{A}$ is a Kaplansky class that is closed under direct limits.

In Section 2 of this paper we study complexes in the class $\mathcal{E} \mathcal{A}^{\perp}$ and the completeness of the cotorsion pair $\left(\mathcal{E} \mathcal{A}, \mathcal{E} \mathcal{A}^{\perp}\right)$. Here $\mathcal{E} \mathcal{A}$ stands for the class of all exact complexes with each term in $\mathcal{A}$. It is shown that $\left(\mathcal{E} \mathcal{A}, \mathcal{E} \mathcal{A}^{\perp}\right)$ is a complete cotorsion pair whenever $(\mathcal{A}, \mathcal{B})$ is a cotorsion pair in $R$-Mod and $\mathcal{A}$ is closed under pure submodules and cokernels of pure monomorphisms. This does not require $\mathcal{A}$ to be closed under direct limits. In addition, some applications are given.

Section 3 is devoted to studying the existence of $\overline{\mathcal{A}}$-covers and $\overline{\mathcal{B}}$-envelopes of special complexes. We prove that each complex of $R$-modules that is bounded above has an $\overline{\mathcal{A}}$-cover and each complex of $R$-modules that is bounded below has a $\overline{\mathcal{B}}$-envelope whenever $\mathcal{A}$ is a covering class and $\mathcal{B}$ is an enveloping class in $R$-Mod.

\section{2. $\mathcal{E} \mathcal{A}$-covers of complexes}

Let $\mathcal{E} \mathcal{A}$ denote the class of all exact complexes $C$ with each term $C^{n}$ in $\mathcal{A}$.

Proposition 2.1. Let $C$ be a complex. Then $C$ is in $\mathcal{E} \mathcal{A}^{\perp}$ if and only if $C^{n}$ is in $\mathcal{A}^{\perp}$ for all $n \in \mathbb{Z}$ and $\mathcal{H o m}(G, C)$ is exact for each $G \in \mathcal{E} \mathcal{A}$.

Proof. Suppose that $(C, \delta)$ is in $\mathcal{E} \mathcal{A}^{\perp}$ and let

$$
0 \longrightarrow C^{n} \stackrel{\alpha}{\longrightarrow} X \longrightarrow F \longrightarrow 0
$$

be an extension in $R$-Mod with $F \in \mathcal{A}$. By the factor theorem (see [2, Theorem 3.6]) we have the following commutative diagram

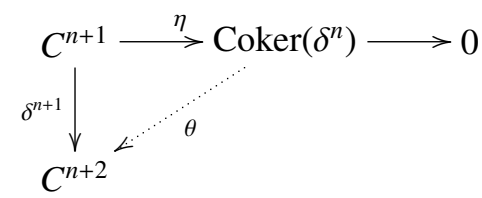

where $\eta: C^{n+1} \rightarrow \operatorname{Coker}\left(\delta^{n}\right)$ is the natural epimorphism. We form the pushout of $C^{n} \stackrel{\alpha}{\longrightarrow} X$ and $C^{n} \stackrel{\delta^{n}}{\longrightarrow} C^{n+1}$ and obtain the following commutative diagram

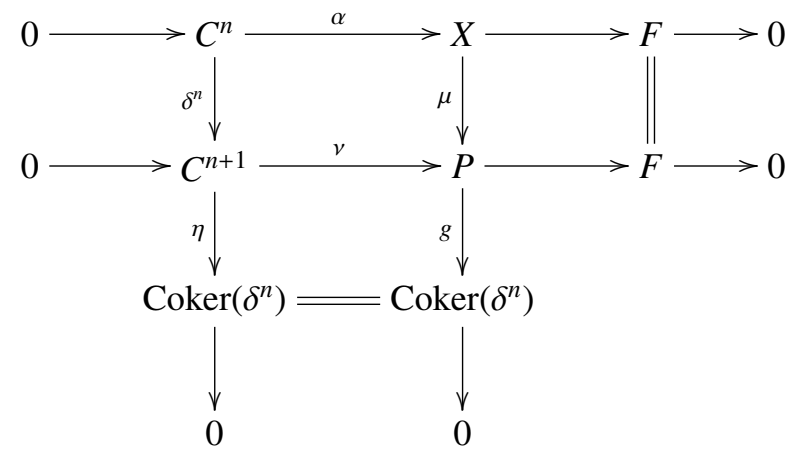


So we have the following commutative diagram

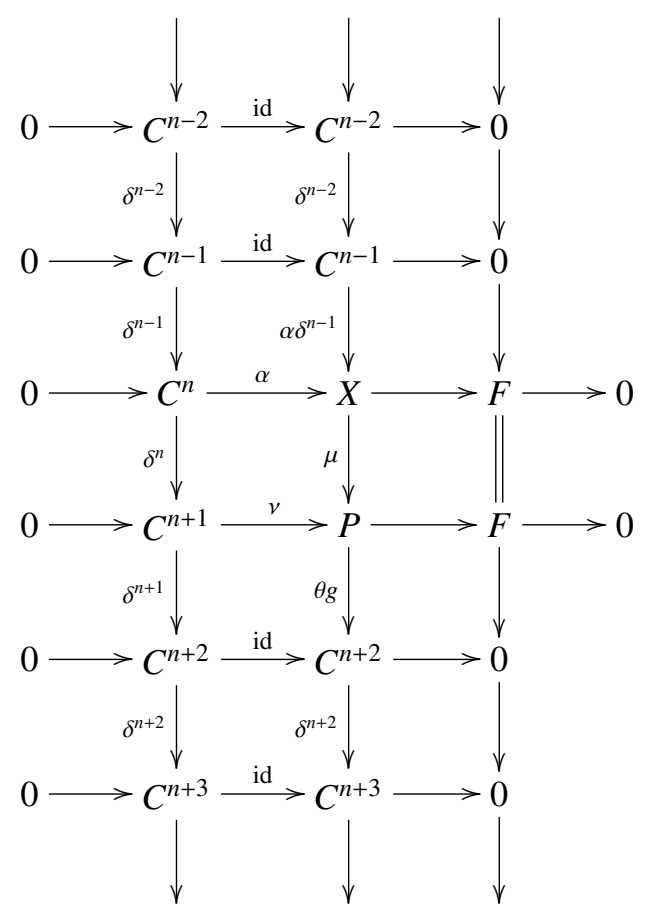

and can form the complex

$$
W=\cdots \longrightarrow C^{n-2} \longrightarrow C^{n-1} \longrightarrow X \longrightarrow P \longrightarrow C^{n+2} \longrightarrow \cdots .
$$

Thus we have an exact sequence of complexes

$$
0 \longrightarrow C \longrightarrow W \longrightarrow \bar{F}[-n-1] \longrightarrow 0 \text {. }
$$

By our hypothesis, the sequence splits in $\mathscr{C}(R)$ and so the sequence

$$
0 \longrightarrow C^{n} \longrightarrow X \longrightarrow F \longrightarrow 0
$$

splits in $R$-Mod. Therefore, $C^{n}$ is in $\mathcal{A}^{\perp}$.

For each $G \in \mathcal{E} \mathcal{A}$ we have that $\mathcal{H} \operatorname{om}(G, C)$ is exact if and only if for each $n$ each map of complexes $f: G \rightarrow C[n]$ is homotopic to 0 . This is equivalent to the requirement that for each $n$ and each map of complexes $f: G \rightarrow C[n]$ the sequence

$$
0 \longrightarrow C[n] \longrightarrow M(f) \longrightarrow G[1] \longrightarrow 0
$$

splits or, equivalently, that for each $n$ and each map of complexes $f: G \rightarrow C[n]$ the sequence

$$
0 \longrightarrow C \longrightarrow M(f)[-n] \longrightarrow G[1-n] \longrightarrow 0
$$

splits where $M(f)$ denotes the mapping cone of $f$. 
Since $G$ is in $\mathcal{E} \mathcal{A}$ we also have $G[1-n]$ in $G \in \mathcal{E} \mathcal{A}$. By our hypothesis we have $\operatorname{Ext}^{1}(G[1-n], C)=0$. So the sequence

$$
0 \longrightarrow C \longrightarrow M(f)[-n] \longrightarrow G[1-n] \longrightarrow 0
$$

splits and $\mathcal{H} \operatorname{om}(G, C)$ is an exact complex.

Suppose that $C^{n}$ is in $\mathcal{A}^{\perp}$ for all $n \in \mathbb{Z}$ and that $\mathcal{H} \operatorname{om}(G, C)$ is exact for each $G \in \mathcal{E} \mathcal{A}$. Each exact sequence

$$
0 \longrightarrow C \longrightarrow W \longrightarrow G \longrightarrow 0
$$

of complexes with $G \in \mathcal{E} \mathcal{A}$ splits at the module level. So this sequence is isomorphic to

$$
0 \longrightarrow C \longrightarrow M(f) \longrightarrow G \longrightarrow 0
$$

where $f: G[-1] \rightarrow C$ is a map of complexes.

Since $\mathcal{H} \operatorname{om}(G[-1], C)$ is exact the sequence

$$
0 \longrightarrow C \longrightarrow M(f) \longrightarrow G \longrightarrow 0
$$

splits in $\mathscr{C}(R)$ by [13, Lemma 2.3.2]. Therefore

$$
0 \longrightarrow C \longrightarrow W \longrightarrow G \longrightarrow 0
$$

also splits and our result is established.

Lemma 2.2. If $G$ is in $\mathcal{A}^{\perp}$, then $\bar{G}[-n]$ is in $\mathcal{E} \mathcal{A}^{\perp}$ for all $n \in \mathbb{Z}$.

Proof. It is enough to prove that $\operatorname{Ext}^{1}(F, \bar{G}[-n])=0$ for each $F \in \mathcal{E} \mathcal{A}$. Let

$$
0 \longrightarrow \bar{G}[-n] \stackrel{\alpha}{\longrightarrow} X \longrightarrow F \longrightarrow 0
$$

be an extension in $\mathscr{C}(R)$ and consider the following commutative diagram

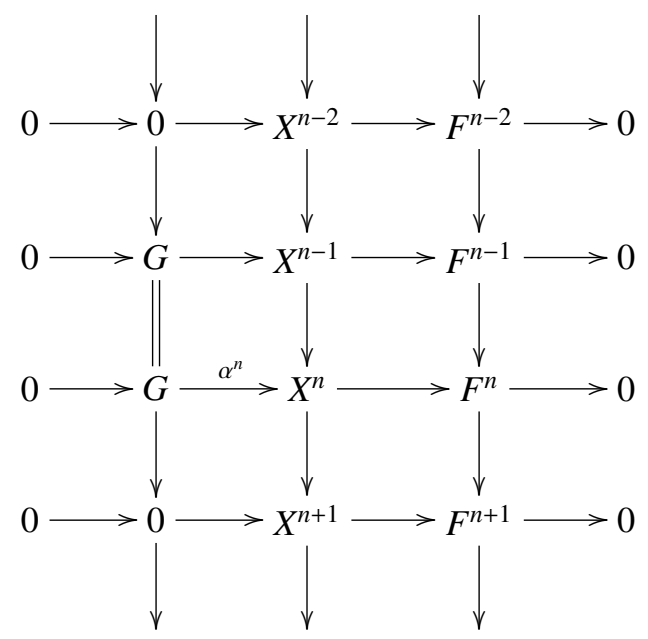


Since $F^{n}$ is in $\mathcal{A}$ and $G$ is in $\mathcal{A}^{\perp}$ we have $\operatorname{Ext}^{1}\left(F^{n}, G\right)=0$. That is, the sequence

$$
0 \longrightarrow G \stackrel{\alpha^{n}}{\longrightarrow} X^{n} \longrightarrow F^{n} \longrightarrow 0
$$

splits in $R$-Mod. So there exists $h^{n}: X^{n} \rightarrow G$ such that $h^{n} \alpha^{n}=1$.

We define $h^{n-1}: X^{n-1} \rightarrow G$ by $h^{n-1}=h^{n} \delta_{X}^{n-1}$ and $h^{i}=0$ for $i \neq n, n-1$. Thus we obtain a map of complexes $h: X \rightarrow \bar{G}[-n]$ such that $h \alpha=1$. So the sequence

$$
0 \longrightarrow \bar{G}[-n] \stackrel{\alpha}{\longrightarrow} X \longrightarrow F \longrightarrow 0
$$

splits in $\mathscr{C}(R)$ and our result is established.

Lemma 2.3. If an injective module I is in $\mathcal{A}$, then $\underline{I}[-n]$ is in $\mathcal{E} \mathcal{A}^{\perp}$ for all $n \in \mathbb{Z}$.

Proof. It is enough to prove that each map $f: F \rightarrow \underline{I}[-n]$ is homotopic to zero for each $F \in \mathcal{E} \mathcal{A}$. Since $f^{n} d^{n-1}=0$ we obtain $Z^{n}(F)=B^{n}(F) \subseteq \operatorname{Ker}\left(f^{n}\right)$ and so the following diagram

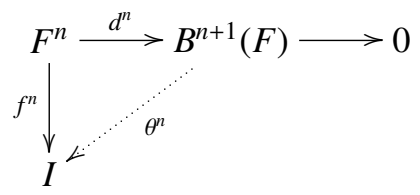

commutes.

Again, since $I$ is injective there exists $S^{n+1}: F^{n+1} \rightarrow I$ such that the diagram

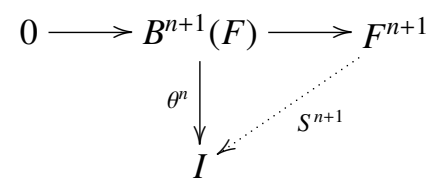

is commutative. Thus $S^{n+1} d^{n}=f^{n}$. That is, the map $f$ is null homotopic and our result follows.

THEOREM 2.4. If $(\mathcal{A}, \mathcal{B})$ is a cotorsion pair in $R$-Mod, then $\left(\mathcal{E} \mathcal{A}, \mathcal{E} \mathcal{A}^{\perp}\right)$ is a cotorsion pair in $\mathscr{C}(R)$.

Proof. It suffices to prove that ${ }^{\perp}\left(\mathcal{E} \mathcal{A}^{\perp}\right) \subseteq \mathcal{E} \mathcal{A}$. If $F \in^{\perp}\left(\mathcal{E} \mathcal{A}^{\perp}\right)$, then $\operatorname{Ext}^{1}(F, C)=0$ for all $C \in \mathcal{E} \mathcal{A}^{\perp}$. For each $n \in \mathbb{Z}$ and each $G \in \mathcal{B}=\mathcal{A}^{\perp}$ let

$$
0 \longrightarrow G \stackrel{\alpha}{\longrightarrow} X \stackrel{\beta}{\longrightarrow} F^{n} \longrightarrow 0
$$


be an extension in $R$-Mod. We consider the following commutative diagram

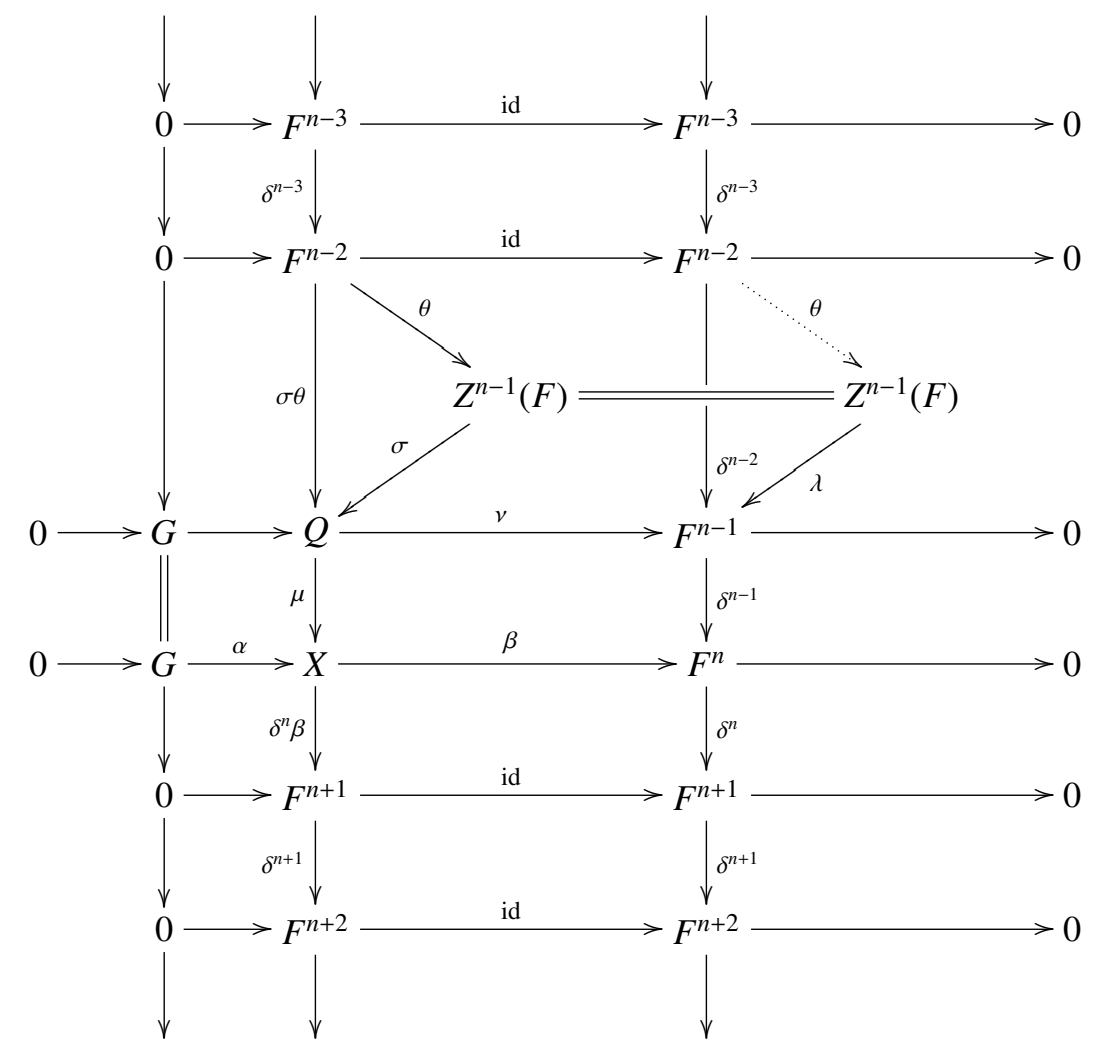

where $\lambda: Z^{n-1}(F) \rightarrow F^{n-1}$ is the natural inclusion and $Q$ is the pullback of $\beta$ and $\delta^{n-1}$.

We get a complex

$$
W=\cdots \longrightarrow F^{n-2} \longrightarrow Q \longrightarrow X \longrightarrow F^{n+1} \longrightarrow \cdots
$$

and an exact sequence

$$
0 \longrightarrow \bar{G}[-n] \longrightarrow W \longrightarrow F \longrightarrow 0
$$

in $\mathscr{C}(R)$.

Since $G$ is in $\mathcal{A}^{\perp}$ we have that $\bar{G}[-n]$ is in $\mathcal{E} \mathcal{A}^{\perp}$ by Lemma 2.2. By the hypothesis $\operatorname{Ext}^{1}(F, \bar{G}[-n])=0$. So the sequence (2.1) splits and the sequence

$$
0 \longrightarrow G \stackrel{\alpha}{\longrightarrow} X \stackrel{\beta}{\longrightarrow} F^{n} \longrightarrow 0
$$

in $R$-Mod splits. Thus $F^{n}$ is in $\mathcal{A}$ for all $n \in \mathbb{Z}$. 
Next we prove that $F$ is exact. Let $f^{n}: F^{n} / B^{n}(F) \rightarrow I$ be an injection with $I$ injective. Then $f^{n}$ induces a map $f: F \rightarrow \underline{I}[-n]$ as follows

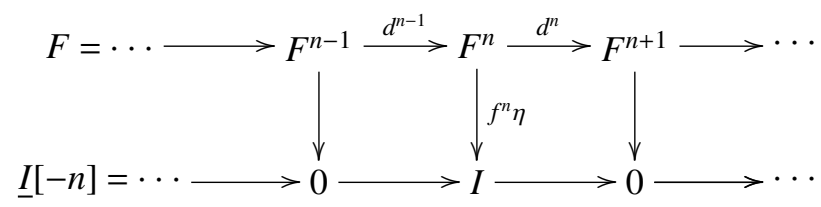

where $\eta: F^{n} \rightarrow F^{n} / B^{n}(F)$ is the natural surjection. By Lemma 2.3, $f$ is homotopic to zero. Let $\left\{S^{n}\right\}$ be the homotopy. Then $S^{n+1} d^{n}=f^{n} \eta$ and so $Z^{n}(F) \subseteq B^{n}(F)$. Thus $F$ is in $\mathcal{E} \mathcal{A}$. Therefore, we may deduce that $\left(\mathcal{E} \mathcal{A}, \mathcal{E} \mathcal{A}^{\perp}\right)$ is a cotorsion pair and our result is established.

Remark 2.5. Proposition 2.1 and Theorem 2.4 are similar to [16, Proposition 3.3] by Gillespie, but our proofs are more direct.

Lemma 2.6. Suppose that $S, T$ and $M$ are modules such that $S \subseteq T \subseteq M$. If $S$ is pure in $M$ and $T / S$ is pure in $M / S$, then $T$ is pure in $M$.

We define the cardinality of a complex $C$ to be $\left|\bigsqcup_{n \in \mathbb{Z}} C^{n}\right|$.

Lemma 2.7 [1, Proposition 4.1]. Let $|R| \leq \boldsymbol{\aleph}$ where $\boldsymbol{\aleph}$ is some infinite cardinal. Then for each $C \in \mathscr{C}(R)$ and each element $x \in C$ (that is, $x \in C^{n}$ for some $n$ ) there exists an exact subcomplex $L \leq X$ such that $x \in L^{k},|L| \leq \boldsymbol{N}$ and $L^{j} \leq C^{j}$ is a pure submodule for all $j \in \mathbb{Z}$.

Theorem 2.8. Let $(\mathcal{A}, \mathcal{B})$ be a cotorsion pair in $R$-Mod. If $\mathcal{A}$ is closed under taking pure submodules and cokernels of pure monomorphisms, then the cotorsion pair $\left(\mathcal{E} \mathcal{A}, \mathcal{E} \mathcal{A}^{\perp}\right)$ is complete.

Proof. Suppose that $G$ is in $\mathcal{E} \mathcal{A}$ and $|R| \leq \boldsymbol{\aleph}$ for some infinite cardinal $\aleph$. We will show that $G$ is equal to the union of a continuous chain $\left(P_{\alpha}\right)_{\alpha<\lambda}$ of exact subcomplexes of $G$ where $\left|P_{0}\right| \leq \boldsymbol{\aleph},\left|P_{\alpha+1} / P_{\alpha}\right| \leq \boldsymbol{\aleph}$ and $P_{\alpha}^{i}$ is pure $G^{i}$ for all $\alpha$ and all $i \in \mathbb{Z}$.

Set $T=\bigsqcup_{n \in \mathbb{Z}} G^{n}$. We may well-order the set $T$ so that for some ordinal $\lambda$

$$
T=\left\{x_{0}, x_{1}, x_{2}, \ldots, x_{\alpha}, \ldots\right\}_{\alpha<\lambda} .
$$

For $x_{0}$ we use Lemma 2.7 to find an exact subcomplex $P_{1} \subseteq G$ containing $x_{0}$ such that $\left|P_{1}\right| \leq \boldsymbol{\aleph}$ and $P_{1}^{i}$ is pure in $G^{i}$ for all $i \in \mathbb{Z}$. Then $G / P_{1}$ is in $\mathcal{E} \mathcal{A}$.

Now $\overline{x_{1}} \in G / P_{1}$. Therefore we can find an exact subcomplex $P_{2} / P_{1} \subseteq G / P_{1}$ containing $\overline{x_{1}}$ such that $\left|P_{2} / P_{1}\right| \leq \boldsymbol{N}$ and $\left(P_{2} / P_{1}\right)^{i}$ is pure in $\left(G / P_{1}\right)^{i}$ for all $i \in \mathbb{Z}$. Then $\left(G / P_{1}\right) /\left(P_{2} / P_{1}\right) \cong G / P_{2}$ is in $\mathcal{E} \mathcal{A}, P_{2}$ is exact and $P_{2}^{i}$ is pure in $G^{i}$ by Lemma 2.6. Note that $P_{1} \subseteq P_{2}$ and $x_{0}, x_{1} \in P_{2}$.

In general, given an ordinal $\alpha$ and having constructed exact subcomplexes $P_{1} \subseteq$ $P_{2} \subseteq \cdots \subseteq P_{\alpha}$ where $x_{\gamma} \in P_{\alpha}$ for all $\gamma<\alpha$, we find an exact subcomplex $P_{\alpha+1} \subseteq G$ as follows. We have $\overline{x_{\alpha}} \in G / P_{\alpha}$ and so by Lemma 2.7 we can find an exact 
subcomplex $P_{\alpha+1} / P_{\alpha} \subseteq G / P_{\alpha}$ containing $\overline{x_{\alpha}}$ such that $\left|P_{\alpha+1} / P_{\alpha}\right| \leq \boldsymbol{\aleph}$ and $\left(P_{\alpha+1} / P_{\alpha}\right)^{i}$ is pure in $\left(G / P_{\alpha}\right)^{i}$ for all $i \in \mathbb{Z}$. Thus $\left(G / P_{\alpha}\right) /\left(P_{\alpha+1} / P_{\alpha}\right) \cong G / P_{\alpha+1}$ is in $\mathcal{E} \mathcal{A}$, whence $P_{\alpha+1}$ is exact and $P_{\alpha+1}^{i}$ is pure in $G^{i}$.

We now have

$$
P_{1} \subseteq P_{2} \subseteq \cdots \subseteq P_{\alpha} \subseteq P_{\alpha+1}
$$

and

$$
x_{0}, x_{1}, \ldots, x_{\alpha} \in P_{\alpha+1} .
$$

In the case where $\alpha$ is a limit ordinal we just define $P_{\alpha}=\bigcup_{\gamma<\alpha} P_{\gamma}$. Then, as we noted above, $P_{\alpha}$ is exact, $x_{\gamma} \in P_{\alpha}$ and $P_{\alpha}^{i}$ is pure in $G^{i}$ for all $i \in \mathbb{Z}$ and all $\gamma<\alpha$. This construction gives us the directed continuous chain $\left(P_{\alpha}\right)_{\alpha<\lambda}$.

If $C$ is a complex such that $\operatorname{Ext}^{1}\left(P_{0}, C\right)=0$ and $\operatorname{Ext}^{1}\left(P_{\alpha+1} / P_{\alpha}, C\right)=0$ whenever $\alpha+1<\lambda$, then $\operatorname{Ext}^{1}(G, C)=0$ by [14, Lemma 4.5]. Let $X$ be a set of representatives of all complexes $G \in \mathcal{E} \mathcal{A}$ with $|G| \leq \boldsymbol{\aleph}$. Then $\mathcal{E} \mathcal{A}^{\perp}=X^{\perp}$. That is, $\left(\mathcal{E} \mathcal{A}, \mathcal{E} \mathcal{A}^{\perp}\right)$ is cogenerated by $X$. Thus $\left(\mathcal{E} \mathcal{A}, \mathcal{E} \mathcal{A}^{\perp}\right)$ is a complete cotorsion pair.

REMARK 2.9. In [16] it was shown that $\left(\mathcal{E} \mathcal{A}, \mathcal{E} \mathcal{A}^{\perp}\right)$ is a complete cotorsion pair whenever $\mathcal{A}$ is a Kaplansky class that is closed under direct limits. In Theorem 2.8 we assume that $\mathcal{A}$ is closed under pure submodules and cokernels of pure monomorphisms. Such a class is automatically a Kaplansky class, but need not be closed under direct limits.

Corollary 2.10. Let $(\mathcal{A}, \mathcal{B})$ be a cotorsion pair in $R$-Mod. If $\mathcal{A}$ is closed under pure submodules, cokernels of pure monomorphisms and direct limits, then the cotorsion $\operatorname{pair}\left(\mathcal{E} \mathcal{A}, \mathcal{E} \mathcal{A}^{\perp}\right)$ is perfect.

According to [10] a module $M$ is called Gorenstein flat if there exists an exact sequence

$$
\cdots \longrightarrow F_{-1} \longrightarrow F_{0} \longrightarrow F_{1} \longrightarrow \cdots
$$

in $R$-Mod of flat $R$-modules such that $M=\operatorname{Ker}\left(F_{0} \rightarrow F_{1}\right)$ and the sequence remains exact whenever $E \otimes-$ is applied, where $E$ is an injective right $R$-module.

Let $\mathcal{G F}$ denote the class of all Gorenstein flat left $R$-modules. In [12, Theorem 3.1.9] (see also [9]) it was proved that over a right coherent ring, $\left(\mathcal{G F}, \mathcal{G F}^{\perp}\right.$ ) is a perfect and hereditary cotorsion pair.

COROLlary 2.11. Every complex over a right coherent ring has an $\mathcal{G} \mathcal{F}$-cover.

Proof. By [12, Corollary 2.1.9] the class $\mathcal{G F}$ is closed under direct limits. Thus it is enough to prove that $\mathcal{G F}$ is closed under pure submodules and cokernels of pure monomorphisms.

Suppose that

$$
0 \rightarrow P \rightarrow M \rightarrow M / P \rightarrow 0
$$

is pure exact in $R$-Mod, where $M \in G \mathcal{F}$. Then

$$
0 \rightarrow(M / P)^{+} \rightarrow M^{+} \rightarrow P^{+} \rightarrow 0
$$


is split and $M^{+} \in \mathcal{G I}$ by [18, Theorem 3.6]. Here $\mathcal{G I}$ denotes the class of Gorenstein injective modules. Thus $(M / P)^{+}$and $P^{+}$are in $\mathcal{G I}$ by [18, Theorem 2.6], which implies that $M / P$ and $P$ are in $\mathcal{G F}$.

We use the symbol $\mathcal{F}_{n}$ to denote the class of all left $R$-modules with flat dimension less than or equal to a fixed nonnegative integer $n$. In [21, Theorem 3.4] it was proved that $\left(\mathcal{F}_{n}, \mathcal{F}_{n}^{\perp}\right)$ is a perfect and hereditary cotorsion pair. Note that $\mathcal{F}_{n}$ is closed under pure submodules, cokernels of pure monomorphisms and direct limits. Thus we have the following result.

Corollary 2.12. Every complex has an $\mathcal{E F}_{n}$-cover.

A left $R$-module $M$ is called min-flat (see [20]) if $\operatorname{Tor}_{1}(R / I, M)=0$ for each simple right ideal $I$. Let $\mathcal{M F}$ denote the class of all min-flat left $R$-modules. In [20, Theorem 3.4] it was proved that $\left(\mathcal{M F}, \mathcal{M F} \mathcal{F}^{\perp}\right)$ is a perfect cotorsion pair. Note that $\mathcal{M F}$ is closed under pure submodules, cokernels of pure monomorphisms and direct limits.

Corollary 2.13. Every complex has an $\mathcal{E} \mathcal{M F}$-cover.

\section{Covers and envelopes of special complexes}

Let $\mathcal{A}$ and $\mathcal{B}$ be classes of $R$-modules. In this section we consider the existence of a $\overline{\mathcal{A}}$-cover of a complex that is bounded above and a $\overline{\mathcal{B}}$-envelope of a complex that is bounded below. Here $\overline{\mathcal{A}}$ and $\overline{\mathcal{B}}$ stand for the classes of all complexes with each term in $\mathcal{A}$ and $\mathcal{B}$, respectively.

LeMma 3.1. Let $C$ be a complex.

(1) If $\varphi: G \rightarrow C$ is an $\overline{\mathcal{A}}$-precover in $\mathscr{C}(R)$, then $\varphi^{n}: G^{n} \rightarrow C^{n}$ is an $\mathcal{A}$-precover in $R$-Mod for all $n \in \mathbb{Z}$.

(2) If $\varphi: C \rightarrow G$ is a $\overline{\mathcal{B}}$-preenvelope in $\mathscr{C}(R)$, then $\varphi^{n}: C^{n} \rightarrow G^{n}$ is a $\mathcal{B}$-preenvelope in $R$-Mod for all $n \in \mathbb{Z}$.

Proof. (1) Let $D$ be in $\mathcal{A}$ and let $f: D \rightarrow C^{n}$ be an $R$-homomorphism. We define a map of complexes $\bar{f}: \bar{D}[-n-1] \rightarrow C$ as follows:

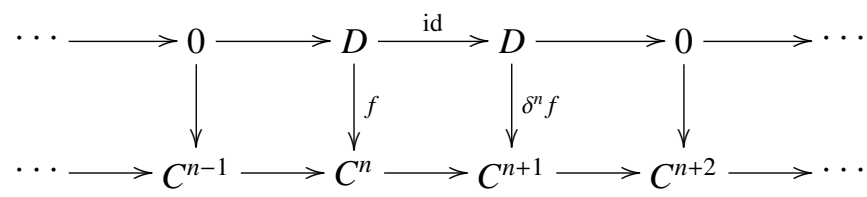

Since $\bar{D}[-n-1]$ is in $\overline{\mathcal{A}}$ there is a map $h: \bar{D}[-n-1] \rightarrow G$ such that $\varphi h=\bar{f}$. So we have a commutative diagram

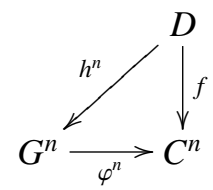

This means that $\varphi^{n}: G^{n} \rightarrow C^{n}$ is an $\mathcal{A}$-precover of $C^{n}$. 
(2) Let $F$ be in $\mathcal{B}$ and let $f: C^{n} \rightarrow F$ be an $R$-homomorphism. We define a map of complexes $\alpha: C \rightarrow \bar{F}[-n]$ as follows:

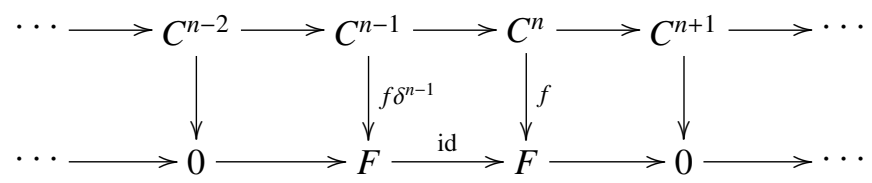

Since $\bar{F}[-n]$ is in $\overline{\mathcal{B}}$ there is a map $\beta: G \rightarrow \bar{F}[-n]$ such that $\varphi \beta=\alpha$. So we have a commutative diagram

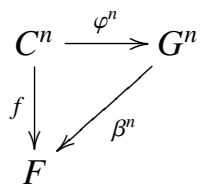

That is, $\varphi^{n}: C^{n} \rightarrow G^{n}$ is a $\mathcal{B}$-preenvelope of $C^{n}$.

TheOREM 3.2. Let $\mathcal{A}$ be a covering class in $R$-Mod and let the complex

$$
C=\cdots \rightarrow C^{-2} \rightarrow C^{-1} \rightarrow C^{0} \rightarrow 0 \cdots
$$

be bounded above. Then:

(1) $C$ has an $\overline{\mathcal{A}}$-cover;

(2) if $\varphi: G \rightarrow C$ is an $\overline{\mathcal{A}}$-cover in $\mathscr{C}(R)$, then $\varphi^{0}: G^{0} \rightarrow C^{0}$ is an $\mathcal{A}$-cover.

Proof. Part (1) follows from some ideas in the proof of [13, Theorem 3.3.10].

(2) We begin by proving that the complex $G$ is bounded. The complex

$$
G^{*}=\cdots \rightarrow 0 \rightarrow G^{0} \rightarrow G^{1} \rightarrow \cdots
$$

is in $\overline{\mathcal{A}}$ and the obvious induced map $G^{*} \rightarrow C$ is an $\overline{\mathcal{A}}$-precover. So $G$ is a direct summand of $G^{*}$ and hence $G$ is bounded above.

Next we prove that $\varphi^{0}: G^{0} \rightarrow C^{0}$ is an $\mathcal{A}$-cover of $C^{0}$. By Lemma 3.1 we know that $\varphi^{0}: G^{0} \rightarrow C^{0}$ is an $\mathcal{A}$-precover of $C^{0}$. Let $\varphi^{0}: G\left(C^{0}\right) \rightarrow C^{0}$ be the $\mathcal{A}$-cover of $C^{0}$ in $R$-Mod. We consider the splitting epimorphism $\beta: G\left(C^{0}\right) \rightarrow C^{0}$ such that $\alpha^{0} \beta=\varphi^{0}$. We take the complex

$$
G^{*}=\cdots \longrightarrow G^{-2} \stackrel{\delta_{G}^{-2}}{\longrightarrow} G^{-1} \stackrel{\beta \delta_{G}^{-1}}{\longrightarrow} G\left(C^{0}\right) \longrightarrow 0 \longrightarrow \cdots
$$

We also consider the map of complexes given by

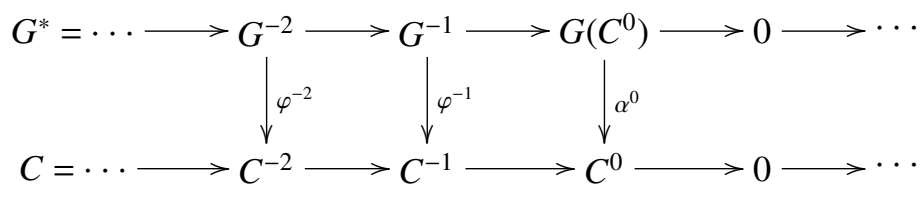


It is easy to check that the above map, which we call $\alpha: G^{*} \rightarrow C$, is an $\overline{\mathcal{A}}$-precover. Thus there exists a splitting epimorphism $g: G^{*} \rightarrow G$ such that $\varphi g=\alpha$. That is, the diagram

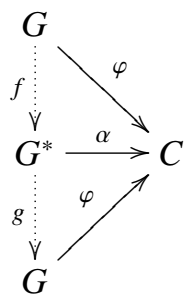

commutes and $g f$ is an automorphism. Hence $\alpha^{0} \beta g^{0}=\varphi^{0} g^{0}=\alpha^{0}$ and so $\beta g^{0}$ is an automorphism, which means that $\varphi^{0}: G^{0} \rightarrow C^{0}$ is an $\mathcal{A}$-cover of $C^{0}$.

Theorem 3.3. Let $\mathcal{B}$ be an enveloping class in $R$-Mod and let the complex

$$
(C, \delta)=\cdots \rightarrow 0 \rightarrow C^{0} \rightarrow C^{1} \rightarrow C^{2} \rightarrow \cdots
$$

be bounded below. Then:

(1) $C$ has a $\overline{\mathcal{B}}$-envelope;

(2) if $\varphi: C \rightarrow G$ is a $\overline{\mathcal{B}}$-envelope, then $\varphi^{0}: C^{0} \rightarrow G^{0}$ is a $\mathcal{B}$-envelope.

Proof. (1) By the hypothesis we may choose a $\mathcal{B}$-envelope $\varphi^{0}: C^{0} \rightarrow G^{0}$. By analogy with the proof of [13, Theorem 3.3.10] we are going to construct a complex

$$
G=\cdots \longrightarrow 0 \longrightarrow G^{0} \longrightarrow G^{1} \longrightarrow \cdots
$$

with each term in $\mathcal{B}$ and a map of complexes $\varphi: C \rightarrow G$ in the following way. For $i<0$ we take $G^{i}=0$ and $\varphi^{i}=0$. For $i=0$ we take the above envelope. Now for $i>0$ we proceed inductively. Suppose that we have constructed

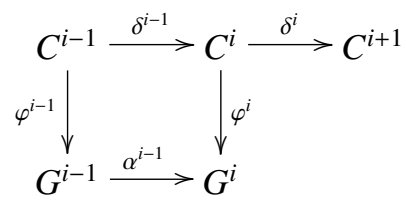

We consider the pushout diagram

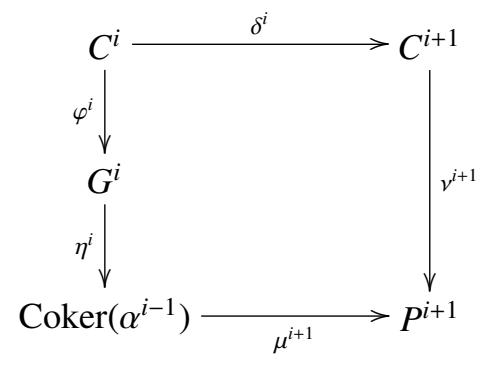


where $\eta^{i}: G^{i} \rightarrow \operatorname{Coker}\left(\alpha^{i-1}\right)$ is the natural epimorphism. Then we take a $\mathcal{B}$-envelope of $P^{i+1}, \beta^{i+1}: P^{i+1} \rightarrow G^{i+1}$. We define $\alpha^{i}: G^{i} \rightarrow G^{i+1}$ to be the composition $\alpha^{i}=\beta^{i+1} \mu^{i+1} \eta^{i}$ and define $\varphi^{i+1}: C^{i+1} \rightarrow G^{i+1}$ by $\varphi^{i+1}=\beta^{i+1} v^{i+1}$. It is not hard to check that this construction gives a complex $G$ with terms in $\mathcal{B}$ and a map of complexes $\varphi: C \rightarrow G$.

Let

$$
F=\cdots \longrightarrow F^{i} \stackrel{\gamma^{i}}{\longrightarrow} F^{i+1} \stackrel{\gamma^{i+1}}{\longrightarrow} F^{i+2} \longrightarrow \cdots
$$

be in $\overline{\mathcal{B}}$ and let $\psi: C \rightarrow F$ be a map of complexes. We are going to construct a morphism of complexes $h: G \rightarrow F$ such that $h \varphi=\psi$. For $i<0$ we take $h^{i}=0$. For $i=0$, since $\varphi^{0}: C^{0} \rightarrow G^{0}$ is a $\mathcal{B}$-envelope, there exists $h^{0}: G^{0} \rightarrow F^{0}$ such that $h^{0} \varphi^{0}=\psi^{0}$. We proceed by induction. Suppose that $h^{i}: G^{i} \rightarrow F^{i}$ is defined such that $h^{i} \varphi^{i}=\psi^{i}$ and $h^{i} \alpha^{i-1}=\gamma^{i-1} h^{i-1}$. By the factor theorem (see [2, Theorem 3.6]) we have the commutative diagram

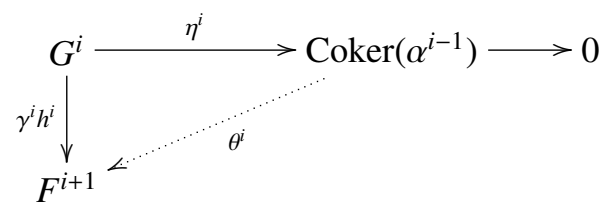

We consider the commutative diagram induced by the pushout

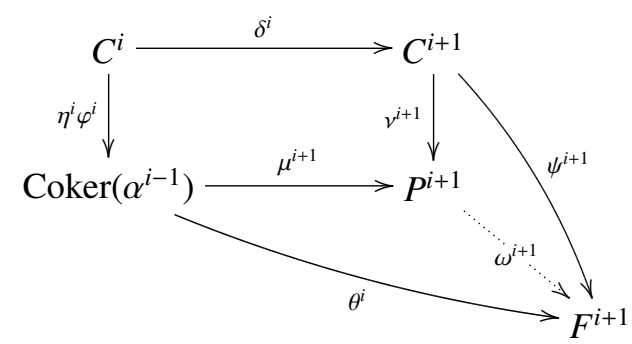

Since $G^{i+1}$ is a $\mathcal{B}$-envelope of $P^{i+1}$ there exists $h^{i+1}: G^{i+1} \rightarrow F^{i+1}$ such that the diagram

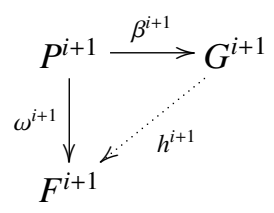

can be completed commutatively. It is easy to see that in this way we obtain a map of complexes $h: G \rightarrow F$ such that $h \varphi=\psi$.

Now let $f: G \rightarrow G$ be a map of complexes such that $f \varphi=\varphi$. For $i<0$ we have $f^{i}=0$. For $i=0$ we know that $f^{0}$ is an automorphism because $\varphi^{0}: C^{0} \rightarrow G^{0}$ is a $\mathcal{B}$-envelope. For $i>0$ we proceed inductively. Suppose that $f^{i-1}$ and $f^{i}$ are automorphisms. 
We show that $f^{i+1}: G^{i+1} \rightarrow G^{i+1}$ is also an automorphism. We consider the commutative diagram

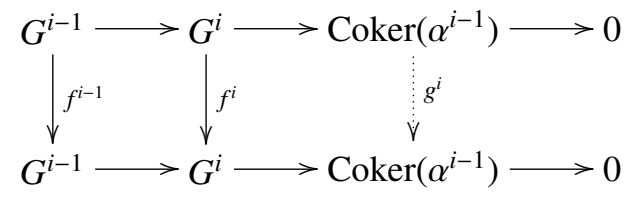

and get that $g^{i}$ is an automorphism. By the properties of a pushout diagram, we get that the diagram

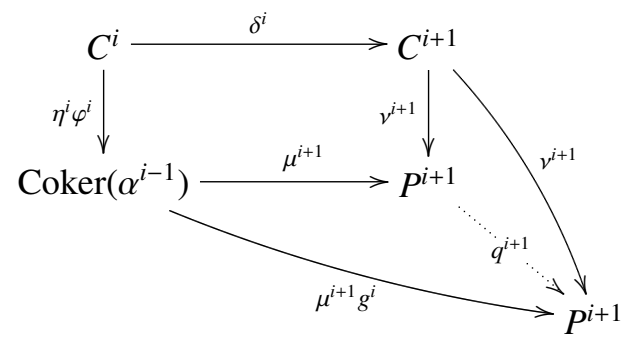

commutes and $q^{i+1}: P^{i+1} \rightarrow P^{i+1}$ is an automorphism. Since $f^{i+1} \alpha^{i}=\alpha^{i} f^{i}$, we get $f^{i+1} \beta^{i+1} \mu^{i+1}=\beta^{i+1} q^{i+1} \mu^{i+1}$ and so we obtain the commutative diagrams

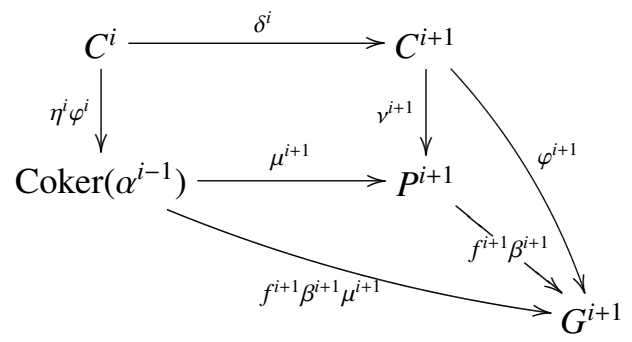

and

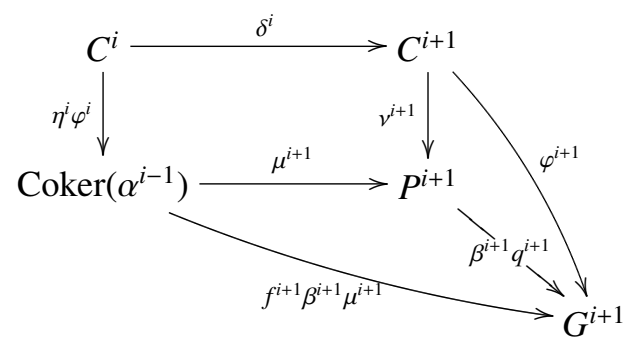

By the properties of pushout diagrams, $f^{i+1} \beta^{i+1}=\beta^{i+1} q^{i+1}$. That is, the diagram

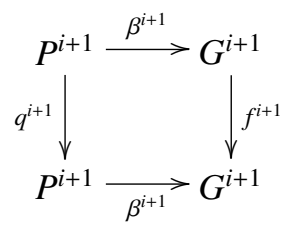


is commutative. Since $\beta^{i+1}: P^{i+1} \rightarrow G^{i+1}$ is a $\mathcal{B}$-envelope and $q^{i+1}$ is an automorphism, it follows that $f^{i+1}$ is an automorphism.

Part (2) follows by an argument like that to prove [13, Proposition 3.2.14].

\section{Acknowledgement}

The authors thank the referee for valuable suggestions and helpful corrections.

\section{References}

[1] S. T. Aldrich, E. E. Enochs, J. R. García Rozas and L. Oyonarte, 'Covers and envelopes in Grothendieck categories: flat covers of complexes with applications', J. Algebra 243 (2001), 615-630.

[2] F. W. Anderson and K. R. Fuller, Rings and Categories of Modules (Springer, Berlin, 1992).

[3] P. C. Eklof and J. Trlifaj, 'How to make Ext vanish', Bull. Lond. Math. Soc. 33 (2001), 41-51.

[4] E. E. Enochs, 'Injective and flat covers, envelopes and resolvents', Israel J. Math. 39 (1981), 189-209.

[5] E. E. Enochs and J. R. García Rozas, 'Tensor products of complexes', Math. J. Okayama Univ. 39 (1997), 17-39.

[6] E. E. Enochs and J. R. García Rozas, 'Gorenstein injective and projective complexes', Comm. Algebra 26 (1998), 1657-1674.

[7] E. E. Enochs and J. R. García Rozas, 'Flat covers of complexes', J. Algebra 210 (1998), 86-102.

[8] E. E. Enochs and O. M. G. Jenda, Relative Homological Algebra (Walter de Gruyter, Berlin, 2000).

[9] E. E. Enochs, O. M. G. Jenda and J. A. López Ramos, 'The existence of Gorenstein flat covers', Math. Scand. 94 (2004), 46-62.

[10] E. E. Enochs, O. M. G. Jenda and B. Torrecillas, 'Gorenstein flat modules', Nanjing Univ. J. Math. Biquarterly 1 (1993), 1-9.

[11] E. E. Enochs, O. M. G. Jenda and J. Xu, 'Orthogonality in the category of complexes', Math. J. Okayama Univ. 38 (1996), 25-46.

[12] E. E. Enochs and J. A. López Ramos, Gorenstein Flat Modules (Nova Science, New York, 2001).

[13] J. R. García Rozas, Covers and Envelopes in the Category of Complexes of Modules (CRC Press, Boca Raton, FL, 1999).

[14] J. Gillespie, 'The flat model structure on $\mathrm{Ch}(R)$ ', Trans. Amer. Math. Soc. 356 (2004), 3369-3390.

[15] J. Gillespie, 'Kaplansky classes and derived categories', Math. Z. 257 (2007), 811-843.

[16] J. Gillespie, 'Cotorsion pairs and degreewise homological model structures', Homology, Homotopy Appl. 10 (2008), 283-304.

[17] R. Göbel and J. Trlifaj, Approximations and Endomorphism Algebras of Modules (Walter de Gruyter, Berlin, 2006).

[18] H. Holm, 'Gorenstein homological dimensions', J. Pure Appl. Algebra 189 (2004), 167-193.

[19] M. Hovey, 'Cotorsion theories, model category structures, and representation theory', Math. Z. 241 (2002), 553-592.

[20] L. X. Mao, 'Min-flat modules and min-coherent rings', Comm. Algebra 35 (2007), 635-650.

[21] L. X. Mao and N. Q. Ding, 'Envelopes and covers by modules of finite FP-injective and flat dimensions', Comm. Algebra 35 (2007), 833-849.

[22] L. Salce, 'Cotorsion theories for abelian groups', in: Symposia Mathematica XXIII. 1979, pp. 11-32.

[23] Z. P. Wang, 'Researches of relative homological properties in the category of complexes', $\mathrm{PhD}$ Thesis, Northwest Normal University, 2010.

[24] J. Z. Xu, Flat Covers of Modules, Lecture Notes in Mathematics, 1634 (Springer, Berlin, 1996). 
ZHANPING WANG, Department of Mathematics, Northwest Normal University, Lanzhou 730070, PR China

e-mail: wangzp@nwnu.edu.cn

ZHONGKUI LIU, Department of Mathematics, Northwest Normal University, Lanzhou 730070, PR China

e-mail: liuzk@nwnu.edu.cn 